\title{
RECOGIENDO EL TESTIGO DE KARL-OTTO APEL. HACIA UNA INTEGRACIÓN DE LA ANTROPOLOGÍA DEL CONOCIMIENTO Y LA PRAGMÁTICA TRASCENDENTAL DEL LENGUAJE*
}

\author{
LAURA MOLINA-MOLINA \\ Universidad de Granada (España) \\ Rheinische Friedrich-Wilhelms - Universität Bonn (Alemania)
}

\begin{abstract}
RESUMEN: la obra del recientemente fallecido filósofo Karl-Otto Apel (15-5-2107) ha pasado por diferentes etapas a lo largo de su desarrollo. Sin embargo, una tarea común a todas ellas ha sido la de reflexionar acerca de aquellas condiciones que a priori son necesarias para conocer. También estas condiciones, así como el modo de concebirlas, han sufrido variaciones a lo largo de sus escritos. $\mathrm{Si}$ en su programa temprano de una antropología del conocimiento aspectos como la reflexión, el cuerpo y el lenguaje tienen un mayor protagonismo, en su posterior pragmática trascendental del lenguaje estos, sin llegar a desaparecer, pasan a reinterpretarse desde la perspectiva de la comunidad de comunicación. Este artículo reconstruye la problemática de los a priori del conocimiento en la filosofía de Apel, y al hacerlo contribuye a una integración, que Apel había planeado llevar a cabo, de esos dos programas.
\end{abstract}

PALABRAS CLAVE: antropología del conocimiento; a priori corporal; a priori lingüístico; comunidad de comunicación; Karl-Otto Apel; pragmática trascendental del lenguaje.

\section{Picking up where Karl-Otto Apel left off Towards an integration of the anthropology of knowledge and the transcendental pragmatics of language}

\begin{abstract}
Karl-Otto Apel (15-5-2107) has gone through different stages throughout its development. However, a common goal of all of them has been to reflect on the conditions that are a priori necessary for knowledge. These conditions, and the way of conceiving them, have undergone variations throughout his writings. While in his early program of an anthropology of knowledge some aspects such as reflection, body and language had a greater role, in his subsequent transcendental pragmatics of language these conditions are reinterpreted, without disappearing, from the perspective of the community of communication. This article reconstructs the problem of the a priori of knowledge in Apel's philosophy and, by doing so, it contributes to an integration, which Apel had planned to achieve, of those two programs.
\end{abstract}

KEY WORDS: anthropology of knowledge, community of communication, corporal a priori, linguistic a priori, Karl-Otto Apel, transcendental pragmatics of language.

Este trabajo forma parte de las actividades del Grupo de Investigación "Conocimiento, verdad y valores" (HUM-432), financiado por la Junta de Andalucía, y de la Unidad Científica de Excelencia FiloLab del Plan Propio de Investigación y Transferencia de la Universidad de Granada (ref.: UCE.PPP2017.04). Ha sido patrocinado por el Programa Ayudas para la Formación del Profesorado Universitario (convocatoria 2013) del Ministerio de Educación, Cultura y Deporte (referencia: FPU13/04478). 
INTRODUCCIÓN

En el año 2005, en el epílogo a la edición francesa de sus escritos Technognomie - eine erkenntnisanthropologische Kategorie (1958) y Das Leibapriori der Erkenntnis (1963), Karl-Otto Apel reconoce la existencia de dos periodos diferentes dentro de su obra filosófica, uno gnoseo-antropológico y otro pragmático-trascendental. Asimismo, considera que la deseada integración entre ambos periodos de cara a una transformación de la filosofía trascendental kantiana aún no ha sido lo suficientemente elaborada, por lo que dedica este escrito a señalar los puntos de conexión entre ambos periodos ${ }^{1}$. La vinculación llevada a cabo por Apel en este epílogo entre el programa gnoseo-antropológico y el pragmático-trascendental tiene lugar en el ámbito de los a priori del conocimiento, señalando con ello que la integración entre ambos periodos filosóficos debería ir, al menos en parte, por ese camino.

En este contexto, lo que el presente artículo ofrece es una reconstrucción de la problemática de los a priori del conocimiento de Apel que contribuya a dicha integración ${ }^{2}$. «Reconstruir» significa aquí mostrar la relación que existe entre los distintos a priori del conocimiento que son tratados en los escritos filosóficos de Apel. Su novedad reside en el hecho de que no se han encontrado escritos de Apel dedicados exclusivamente al tratamiento de esta cuestión, hallándose las reflexiones en torno a la misma diseminadas a lo largo de toda su obra. Una excepción a esta afirmación la encontramos en su entrevista de 1991 con L. Sáez, en la que Apel realiza una serie de consideraciones relativas a la relación existente entre los $a$ priori del cuerpo, la reflexión y el lenguaje ${ }^{3}$. En cuanto a la literatura secundaria, la bibliografía disponible en torno a los a priori del conocimiento en el pensamiento de Apel se centra en otros aspectos de esta problemática y no tiene como objetivo una reconstrucción del tipo que aquí se ofrece ${ }^{4}$. Por otro lado, el interés de una

1 Cf. Apel, K. O., «Postface trente-huit ans après», en: Simonelli, T., L'«a priori» du corps dans le problème de la connaissance. Suivi de Technognomie et de Postface trente-huit ans après, Les Éditions du Cerf, Paris, 2005, 87-8.

2 A favor de una conexión entre ambos programas se muestra R. Maliandi, que considera que la pragmática trascendental del lenguaje, elaborada con el importante objetivo de superar el paradigma de la conciencia, no representa un abandono sino un complemento de la concepción apeliana del a priori corporal (cf. MaLIANDI, R., «El apriori corporal en Apel», en: Rovaletti, M. L. (ed.), Corporalidad. La problemática del cuerpo en el pensamiento actual, Lugar Editorial, Buenos Aires, 1998, 161-77). También H. Nolte pone en conexión la competencia comunicativa con el a priori corporal en el marco de los planteamientos antropológicos tanto de Habermas como de Apel, reconociendo las ventajas que ofrece el planteamiento de este último como un intento adecuado de incorporar el ineludible compromiso corporal al proceso de reflexión (cf. NolTE, H., «Kommunikative Kompetenz und Leibapriori: Zur philosophischen Anthropologie von Jürgen Habermas und Karl-Otto Apel», en: Archiv für Rechts- und Sozialphilosophie, vol. 70, 4, 1984, 518-39).

3 Cf. Apel, K. O., "“A priori de la facticidad” y "a priori de la idealización”. Opacidad y transparencia» (entrevista con L. Sáez Rueda), en: Blanco, D. et al. (eds.), Discurso y Realidad. En debate con K.-O. Apel, Trotta, Madrid, 1994, 251-70.

4 La interesante reconstrucción de los a priori gnoseo-antropológicos que el trabajo de J. Klüver ofrece se centra, sobre todo, en la relación que estos guardan con los diversos 
reconstrucción de este tipo radica en que permitiría mitigar algunos déficits que presentan los últimos desarrollos de la obra apeliana, como por ejemplo la ausencia del elemento corporal, que tantas veces se le ha criticado, como se verá a continuación.

La antropología del conocimiento contiene nociones, como la del a priori corporal, que han resultado muy atractivas para los estudiosos de Apel. Este hecho se debe a la excesiva importancia que el pensamiento apeliano otorga a la reflexión teórica, lo que provoca que el tema de la corporalidad esté implicado en gran parte de los problemas con los que Apel y sus continuadores se enfrentan en la actualidad. A modo de ejemplo, D. Blanco señala el a priori corporal como una de las insuficiencias de la ética apeliana ${ }^{5}$. También en el ámbito de la ética del discurso, A. Cortina propone como complementación de la misma una «antropología axiológica» de la experiencia moral, que incluya sentimientos y valores ${ }^{6}$. Por su parte, J. Conill diagnostica un déficit experiencial en el tratamiento apeliano del cuerpo, excesivamente ligado a las funciones de la conciencia y del lenguaje, e intenta proseguir la antropología del conocimiento de Apel en forma de una antropología de la experiencia, más allá del primado de la epistemología ${ }^{7}$. En contrapartida, en el ámbito de la teoría consensual de la verdad, J. A. Nicolás observa que la recuperación de la evidencia fenomenológica implica una recuperación implícita de la corporalidad como acceso a esa evidencia ${ }^{8}$.

A la hora de desarrollar e interpretar las tesis contenidas tanto en la entrevista de 1991 como en el epílogo de 2005, la reconstrucción que aquí se realiza toma como punto de partida las reflexiones gnoseo-antropológicas llevadas a cabo por Apel. Estas se encuentran, por un lado, en aquellos textos de juventud dedicados íntegramente a exponer su proyecto gnoseo-antropológico y que no han sido incorporados a su obra principal Transformación de la filosofía ${ }^{9}$ (en adelante, $T F$ ) y, por otro, en aquellos

tipos de ciencia que Apel distingue en el marco de su teoría de la ciencia (cf. KLÜver, J., "Transzendentalphilosophie versus Wissenschaftskritik. Rekonstruktion und Kritik des erkenntnisanthropologische Aprioris», en: Kuhlmann, W. / Böhler, D. (eds.), Kommunikation und Reflexion. Zur Diskussion der Transzendentalpragmatik. Antworten auf K.-O. Apel, Suhrkamp, Frankfurt a. M., 1982, 463-99). Si bien no explora suficientemente la conexión entre el programa gnoseo-antropológico y el pragmático-trascendental, el trabajo de A. Centeno representa una importante reconstrucción y delimitación del primero con respecto al segundo (cf. Centeno, A., La antropología del conocimiento en el pensamiento de Karl-Otto Apel. Tesis doctoral publicada en el Repositorio Institucional de la Universidad de Granada DIGIBUG, 2012).

5 Cf. Blanco, D., «El a priori corporal: insuficiencia de la ética discursiva», en: Blanco, D. et al. (eds.), Discurso y realidad. En debate con K.-O. Apel, Trotta, Madrid, 1994, 46-74.

6 Cf. Cortina, A., Ética sin moral, Tecnos, Madrid, 1990.

7 Cf. Conill, J., "Zu einer anthropologischen Hermeneutik der erfahrenden Vernunft», en: Zeitschrift für philosopische Forschung 47/3, 1993, 422-33 e id., «Hermenéutica genealógica desde el cuerpo», en: Estudios Nietzsche, 9, 2009, 15-23.

8 Cf. Nicolás, J. A., "Teoría de la verdad consenso-evidencial y teoría de la verdad fenomenológico-real», en: Blanco, D. et al. (eds.), op. cit., 144-56.

9 Se trata de contribuciones escasamente conocidas que ofrecen, no obstante, una lectura complementaria y diferente de la transformación filosófica descrita en su obra principal y de las exégesis usuales de su pensamiento. Ellas abarcan algunos de los trabajos filosóficos publicados durante la década de los años 50 y 60 (APEL, K. O., «Technognomie 
escritos de juventud que solo exhiben de manera dispersa nociones y reflexiones gnoseo-antropológicas pero que sí forman parte de su obra principal ${ }^{10}$.

El primer apartado de este artículo está dedicado a presentar de manera esquemática una tipología de los distintos a priori del conocimiento que Apel menciona a lo largo de su obra, a fin de introducir el contexto en el que se enmarca la reconstrucción que aquí se ofrece. Seguidamente se exponen e interpretan en un segundo apartado las tesis anunciadas en la entrevista de 1991 tomando como referencia sus consideraciones gnoseo-antropológicas para, en un tercer momento, analizar el contenido del epílogo de 2005. La reinterpretación de la relación existente entre los a priori gnoseo-antropológicos del cuerpo, la reflexión y el lenguaje tomando como referencia el a priori pragmático-trascendental de la comunidad de comunicación se presenta en el penúltimo apartado como una posible vía de integración entre ambos programas. El último apartado del artículo estará dedicado a recoger de manera sucinta las principales conclusiones en las que este trabajo desemboca.

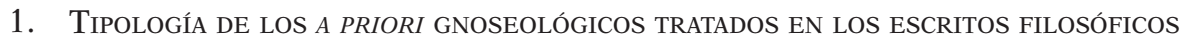
DE APEL

El concepto de «a priori del conocimiento» (Erkenntnisapriori) está presente a lo largo de toda la obra filosófica de Apel y juega un papel fundamental en la medida en que constituye un recurso frecuentemente utilizado por el autor a la hora de distinguir y caracterizar sus propuestas filosóficas frente a las de otros autores y corrientes.

Sin embargo, la importancia de esta noción contrasta con el hecho de que aún no se han encontrado escritos de Apel dedicados a exponer de manera sistemática la denominación, naturaleza, tipología e interrelación de los diferentes a priori a los que él alude en numerosas ocasiones y en contextos de discusión muy diferentes. Además, dependiendo de la temática tratada o del interlocutor al que se esté dirigiendo en cada caso, Apel utiliza distintos términos para referirse al mismo a priori. A continuación se ofrece de manera esquemática una tipología de los principales a priori del conocimiento que Apel menciona a lo largo de su

- eine erkenntnisanthropologische Kategorie», en: FunKe, G. (ed), Konkrete Vernunft, Festschrift für E. Rothacker, Bonn, 1958, 61-79; id., "Kann es ein wissenschaftliches "Welt-Bild" überhaupt geben? Die Theoretische Wissenschaft der Gegenwart in erkenntnisanthropologischer Sicht», en: Zeitschrift für philosophische Forschung, XVI, 1962a, 26-57; id., «Das Leibapriori der Erkenntnis. Eine Erkenntnisanthropologische Betrachtung im Anschluss an Leibnizens Monadenlehre», en: Archiv für Philosophie, 12, 1963, 152-72 e $i d$., "Die erkenntnisanthropologische Funktion der Kommunikationsgemeinschaft und die Grundlagen der Hermeneutik», en: Moser, S. (ed.), Information und Kommunikation, München / Wien, 1968a, 163-71).

10 Apel, K. O., La transformación de la filosofía, 2 vols., Taurus, Madrid, 1985. Centeno (op. cit., 14) afirma que la elaboración de este programa comprende el periodo que va desde mediados de los años 50 hasta mediados de los años 60. Sin embargo, la existencia de escritos posteriores a 1965 en los que Apel sigue tratando cuestiones gnoseo-antropológicas obliga a extender dicha franja. 
obra, ordenados según el programa filosófico al que pertenecen. Entre paréntesis se indica el año de los textos en los que cada uno aparece ${ }^{11}$ :

\begin{tabular}{|c|c|c|c|}
\hline \multicolumn{2}{|c|}{ ANTROPOLOGÍA DEL CONOCIMIENTO } & $\begin{array}{c}\text { PRAGMÁTICA } \\
\text { TRASCENDENTAL } \\
\text { DEL LENGUAJE }\end{array}$ \\
\hline $\begin{array}{c}\text { Leib } \\
\left(1958 ; 1959^{12} ; 1962 \mathrm{a} ;\right. \\
1962 \mathrm{~b}^{13} ; 1963 \mathrm{y} \\
\left.1968 \mathrm{~b}^{14}\right)\end{array}$ & $\begin{array}{c}\text { Reflexion } \\
(1958 ; 1963 \mathrm{y} \\
\left.1972^{15}\right)\end{array}$ & $\begin{array}{c}\text { Sprache } \\
(1968 \mathrm{~b})\end{array}$ & $\begin{array}{c}\text { Kommunikations- } \\
\text { gemeinschaft } \\
\left(1968 \mathrm{a} \text { y } 1970^{16}\right)\end{array}$ \\
\hline $\begin{array}{c}\text { Erkenntnisinteressen } \\
(1958 ; 1968 \mathrm{~b} ; 1970 \mathrm{y}\end{array}$ & $\begin{array}{c}\text { Bewußtsein- } \\
\text { überhaupt } \\
1972)\end{array}$ & Muttersprache & $\begin{array}{c}\text { Argumentations- } \\
\text { gemeinschaft }\end{array}$ \\
$(1962 \mathrm{a}$ y 1968b) & $(1958 \mathrm{y} 1959)$ & $(1972)$ \\
\hline
\end{tabular}

Como puede observarse, tanto los a priori del cuerpo y de la reflexión ${ }^{18}$ como el

11 Salvo aquellos casos específicamente indicados, los años hacen referencia a escritos ya citados.

12 APEL, K. O., «Der philosophische Wahrheitsbegriff einer inhaltlich orientierten Sprachwissenschaft», en: H. Gipper (ed.), Sprache-Schlüssel zur Welt, Festschrift für L. Weisgerber, Düsseldorf, 1959, 11-38 (recopilado en TF I, 101-31).

13 Apel, K. O., "Reflexion und materielle Praxis: zur erkenntnisanthropologischen Begründung der Dialektik zwischen Hegel und Marx», en: Hegel Studien, 1, 1962, 151-66 (recopilado en $T F$ II, 9-26).

14 APEL, K. O., «Szientistik, Hermeneutik, Ideologiekritik: Entwurf einer Wissenschaftlehre in erkenntnisanthropologischer Sicht», en: Wiener Jahrbuch für Philosophie, I, 1968b, 15-45 (recopilada en TF II, 91-120).

15 Se corresponde con la «Introducción» de TF I, 9-72.

16 ApEL, K. O., «Wissenschaft als Emanzipation?», en: Zeitschrift für Allgemeine Wissenschaftstheorie, I, 1970, 173-95 (recopilado en TF II, 121-45).

17 Si bien es cierto que el programa gnoseo-antropológico de Apel recibe una fuerte influencia del planteamiento antropológico de J. Habermas, también lo es el hecho de que ambos enfoques difieren entre sí en aspectos importantes (por ejemplo, en lo que respecta al alcance trascendental del mismo). En relación a esta temática, merecen especial atención los siguientes trabajos: DallmaYr, F., "Critical Theory Criticized: Habermas' Knowledge and Human Interests and its Aftermath», en: Philosophy of the Social Sciences, 2, 1972, 211-29; Lennhardt, Ch., "Rise and Fall of Transcendental Anthropology», en: Philosophy of Social Sciences, 2, 1972, 231-46 y Niquet, M., «Die Identitäten des Menschen. Von der klassischen philosophischen Anthropologie zur Diskursanthropologie», en: ApEL, K. O. / Niquet, M. (eds.), Diskursethik und Diskursanthropologie, 2, Alber, Freiburg, 2002, 95-282.

18 Asumiendo la tradición fenomenológica, Apel distingue entre el cuerpo como Leib y el cuerpo como Körper. El primero hace referencia al cuerpo propio que, no pudiendo ser observado, hace posible la observación de otros cuerpos. El segundo engloba al resto de cuerpos, que sí pueden ser objeto de observación (cf. Apel, K. O., "Comment peut-on thématiser les conditions, non objectivables, de la possibilité de 1'objectivation - celle du corps (L) par exemple?», en: Variations sur l'object: en hommage à Gérard Deledalle, 5, 1/2, 
de los intereses cognoscitivos son tematizados por Apel desde el principio (1958), algo que no ocurre en el caso del a priori de la comunidad de comunicación. Por su parte, el cuerpo deja de ser tratado a partir del mismo año en el que el a priori de la comunidad de comunicación realiza su primera aparición en el marco de las reflexiones gnoseo-antropológicas de Apel (1968). Este último a priori será, de hecho, el único que sobreviva en el programa pragmático-trascendental.

La gnoseo-antropología es una teoría del conocimiento que reconoce como $a$ priori gnoseológicos no solo la conciencia o reflexión del sujeto, como es el caso de la teoría clásica del conocimiento de autores como Platón, Descartes o Kant, sino también el cuerpo del ser-ahí-en-el-mundo ${ }^{19}$. Para Apel, la dimensión corporal del conocimiento humano denota la naturaleza perspectivista y finita del mismo, y esta dimensión del conocimiento guarda, como se verá más adelante, una estrecha vinculación con el hecho de que toda reflexión se lleva a cabo mediante el lenguaje. Por su parte, en la pragmática trascendental del lenguaje, los a priori del cuerpo y de la reflexión ceden su sitio al a priori de la comunidad de comunicación, si bien ello no significa que dejen de estar presentes.

Me propongo mostrar cómo los a priori gnoseo-antropológicos del cuerpo y de la reflexión son integrados en el a priori pragmático-trascendental de la comunidad de comunicación, abriendo así una posible vía de integración entre la antropología del conocimiento y la pragmática trascendental del lenguaje.

En este intento de reconstrucción sistemática me centraré principalmente en los a priori del cuerpo, la reflexión, el lenguaje y la comunidad de comunicación, que son los que Apel menciona cuando señala el vínculo que existe entre su programa gnoseo-antropológico y el pragmático-trascendental.

\section{LA ENTREVISTA DE 1991 A LA LUZ DE LOS ESCRITOS GNOSEO-ANTROPOLÓGICOS}

Como ya se ha mencionado, antes de proceder a analizar el contenido del epílogo de 2005, cabe prestar atención a algunas de las ideas que Apel bosqueja durante la entrevista que mantuvo con L. Sáez en el año 1991. Se trata de una serie de consideraciones esquemáticas, aunque fundamentales, en torno a la naturaleza y relación de los a priori del conocimiento.

$\mathrm{Al}$ referirse al modo como influye el cuerpo en la reflexión, el autor afirma que

ISSS, Vienna, 1993, 11-24). Apel combina además el tratamiento fenomenológico del cuerpo propio con el planteamiento antropológico de H. Plessner, concibiéndolo como un a priori céntrico, frente al a priori de la reflexión, de naturaleza excéntrica. La reflexión, frente al carácter céntrico del cuerpo, es un a priori excéntrico en el sentido de que, gracias al mismo, el ser humano es capaz de distanciarse de la perspectiva desde la que entiende el mundo natural y social.

${ }_{19}$ El trabajo de C. Fabbrizi presenta una visión de la teoría del conocimiento de Kant diferente a la tradicional y explora la noción de cuerpo y su relación con la mente tanto en la etapa pre-crítica de este autor como en su pensamiento crítico (FABBrizi, C., Mente e corpo in Kant, Aracne Editrice, Roma, 2008). 
en su gnoseo-antropología hizo jugar al a priori corporal de forma polar ${ }^{20}$ frente al a priori de la reflexión pero que, más tarde, le asaltó el pensamiento de si este último no sería ya dependiente del primero. En efecto, afirma Apel, «aun cuando las teorías constituyan triunfos del a priori de la reflexión sobre el a priori corporal, estas siguen estando formuladas en un lenguaje en el que se oculta de nuevo el $a$ priori corporal ${ }^{21}$. En otras palabras, dado que el medio en el que el ser humano lleva a cabo sus reflexiones es el lenguaje y en la medida en que todo lenguaje humano introduce una determinada perspectiva finita, las construcciones teóricas fruto de la reflexión se hallan sujetas en última instancia a una perspectiva corporal. A juicio de Apel, este hecho, lejos de ser un obstáculo, constituye más bien una condición posibilitadora, ya que "si no existiese un a priori corporal no se podría adoptar perspectiva alguna ni hacer experimentos, ni siquiera reflexionar» ${ }^{22}$.

Las ideas recogidas en esta parte de la entrevista se comprenden mejor a la luz de algunos de los escritos que Apel dedica a su programa gnoseo-antropológico. Me propongo a continuación desarrollar e interpretar esas ideas a partir de los mismos.

\subsection{Cuerpo y reflexión: una relación de complementariedad}

En primer lugar, en relación a esa «tensión polar» que Apel establece en este programa entre el a priori de la reflexión y el a priori corporal, el autor defiende que dicha tensión nunca desaparece, si bien es cierto que, dependiendo de si nos encontramos ante una experiencia pre-reflexiva o bien ante una reflexión teórica, se da un triunfo o primacía de un a priori con respecto al otro.

Con la expresión «triunfo del a priori de la reflexión sobre el a priori corporal», Apel se refiere a la capacidad del ser humano para asumir u objetivar reflexivamente aquellas condiciones desde las cuales este observa y conoce, con vistas a elaborar teorías cuya validez tenga alcance intersubjetivo o universal. Respecto a la experiencia, la primacía del a priori del cuerpo sobre el de la reflexión significa que, en este caso, los resultados cognitivos a que da lugar dicha experiencia no son producto de una toma de conciencia ni objetivación de las condiciones en las que el ser humano experimenta su entorno. En relación a esto, Apel afirma lo siguiente:

(...) el a priori corporal del conocimiento se encuentra en una relación de complementariedad con el a priori de la conciencia; es decir, en el conjunto del conocimiento ambas condiciones de posibilidad se complementan mutuamente de un modo necesario, pero en la actual realización del conocimiento o bien domina el a priori corporal o bien el a priori de la conciencia: «conocimiento mediante reflexión» y "conocimiento mediante compromiso» se oponen entre sí polarmente (...). Toda experiencia (...) es primariamente conocimiento mediante

20 Apel no llega a explicar qué entiende por «polaridad» o «tensión polar» entre el cuerpo y la reflexión. Atendiendo a los diversos contextos en los que tales expresiones aparecen, se podría decir que para Apel ambos a priori se hayan enfrentados en el sentido de que no pueden disfrutar simultáneamente del mismo protagonismo. El modo en que ambos a priori se comportan según los diferentes tipos de experiencia se verá más claramente a lo largo de los siguientes epígrafes.

21 APEL, K. O., «“A priori de la facticidad”...», op. cit., 269.

22 Ibidem. 
compromiso corporal; toda construcción teórica es primariamente conocimiento mediante reflexión ${ }^{23}$.

La idea de que las construcciones teóricas constituyen un triunfo del a priori de la reflexión con respecto al a priori corporal queda especialmente ejemplificada en aquellas ocasiones en las que Apel reflexiona en torno a las implicaciones gnoseoantropológicas de la teoría de la relatividad o de la mecánica cuántica. A juicio de Apel, estas teorías intentan controlar reflexivamente, a través de una teoría matemática, el a priori corporal, tradicionalmente olvidado por la teoría clásica del conocimiento. Este a priori corporal viene representado en la teoría de la relatividad por el «sistema de referencia» y en la mecánica cuántica por el «acto de medir» del observador a través de un aparato de medición. De este modo, la relevancia gnoseo-antropológica de la Física contemporánea reside, a juicio de Apel, en la toma de conciencia acerca del papel que el cuerpo del observador juega en los resultados de la observación ${ }^{24}$.

\subsection{El vínculo interno entre lenguaje y cuerpo, condición de posibilidad de la reflexión}

En segundo lugar, la tesis según la cual en el lenguaje se oculta siempre el a priori corporal también está presente en escritos anteriores. Con esta última expresión, Apel no quiere decir que el cuerpo se esconda detrás del lenguaje, sino más bien que el lenguaje introduce una perspectiva corporal, finita, señalando así la existencia de un vínculo especial entre ambos a priori.

Para este autor, «la lengua es el medio único e insustituible a través del cual el pensamiento excéntrico se integra siempre de nuevo con las visiones del mundo ligadas a una perspectiva y a un cuerpo $»^{25}$. Esta misma idea viene expresada en su afirmación de que «la mediación de la conciencia por la praxis se produce en el lenguaje ${ }^{26}$. En un texto posterior a $T F$, Apel afirma lo siguiente: «a favor del arraigamiento del lenguaje en la finitud del ser-ahí humano habla también el nexo interno entre el a priori del lenguaje y el a priori corporal. Según la doctrina de Tomás de Aquino y Dante Alighieri los ángeles no necesitan lenguaje ${ }^{27}$.

Finalmente, la tesis de que toda reflexión requiere la mediación de una perspectiva corporal para que pueda llegar a realizarse aparece formulada de distintas maneras en diversos textos de temática gnoseo-antropológica.

23 Cf. Apel, K. O., TF II, 94.

24 Cf. Apel, K. O., «Technognomie...», «Kann es ein wissenschaftliches...», «Das Leibapriori..» y TF II: 91-120, op. cit. M. Katsumori realiza una interesante reflexión acerca del papel que juega la noción de «complementariedad» de N. Bohr en el programa gnoseoantropológico de Apel (cf. Katsumori, M., Niels Bohr's Complementarity: Its Structure, History, and Intersections with Hermeneutics and Deconstruction, Springer Science \& Business Media, 2011, 109-13).

25 APEL, K. O., TF I, 126.

26 APEL, K. O., TF II, 22.

27 Apel, K. O., «¿Es la muerte una condición de posibilidad del significado?», en: ApEL, K. O., Paradigmas de filosofía primera, Prometeo Libros, Buenos Aires, 2013, 94-5. En relación a este punto, Centeno considera que la antropología del conocimiento no tiene en cuenta el giro lingüístico, en el sentido de que no considera al lenguaje el lugar eminente donde plantear específicamente los problemas filosóficos (CENTENo, A., op. cit., 140). Sin embargo, afirmaciones como las anteriores ponen en entredicho la tesis de Centeno. 
Apel considera que el sentido del mundo, su significatividad, nunca puede surgir ante una conciencia pura, que en sí misma es no creativa y vacía de contenido, sino que tiene que estar mediada necesariamente por un compromiso corporal del hombre con su entorno, a través del comercio sensorial, técnico-instrumental o lingüístico que el hombre establece con el mundo a fin de ver satisfechas sus necesidades, intereses y aspiraciones ${ }^{28}$. Además, en un escrito posterior, Apel sostiene que dicha mediación corporal no solo es necesaria para la constitución de sentido, es decir, para que algo se nos presente «como algo», sino también para la justificación de la validez intersubjetiva de nuestros enunciados. Esto significa que el cuerpo, lejos de ser un obstáculo, es condición de posibilidad de la validez intersubjetiva de nuestro conocimiento ${ }^{29}$.

\subsection{Reflexión y cuerpo frente al lenguaje: una relación de dependencia}

En la entrevista de 1991, Apel refuerza la defensa de las tesis señaladas en el epígrafe anterior al afirmar que «tanto el a priori de la reflexión como el a priori corporal pertenecen al a priori lingüístico del conocimiento» ${ }^{30}$. Tomando como referencia algunas de las ideas desarrolladas en los escritos gnoseo-antropológicos de Apel, hay que entender la «relación de pertenencia» que estos dos a priori guardan con respecto al a priori del lenguaje como una relación de dependencia ${ }^{31}$.

A favor de esta interpretación habla, por un lado, lo que Apel denomina la «problemática gnoseo-antropológica del marco», según la cual la imagen del mundo (Weltbild) transmitida a través de la lengua materna constituye el primer y último marco en base al que se constituye y fundamenta toda imagen del mundo humana en general, ya sea filosófica, científica, religiosa o artística ${ }^{32}$. En el caso de los últimos desarrollos científicos de la Física contemporánea, esta problemática se materializa en el hecho de que los lenguajes a través de los que son elaboradas las construcciones teóricas propias de la teoría de la relatividad o de la mecánica cuántica presuponen ya siempre el lenguaje figurativo de la Física clásica ${ }^{33}$.

Por otro lado, su afirmación de que «la lengua constituye el órgano más antiguo y fundamental de la Tecnognomía ${ }^{34}$ también avala la idea de que tanto el a priori de la reflexión como el a priori corporal dependen en última instancia del a priori del

28 Cf. ApEL, K. O., «Technognomie...», op. cit. y TF II, 9-26.

29 Cf. APEL, K. O., TF II, 91-120.

30 Apel, K. O., "“A priori de la facticidad”...», op. cit., 270.

31 Ortiz de Landázuri habla también acerca de la dependencia del doble a priori somático y reflexivo apeliano con respecto a unos juegos del lenguaje enraizados a su vez en la Lebenswelt (cf. ORTIZ DE LANDÁzURI, C., «Los a prioris Antropológicos del Conocimiento», Thémata. Revista de filosofía, No. 23, 1999, 285).

32 Cf. Apel, K. O., «Technognomie...», op. cit. La misma idea queda reflejada en otro momento, cuando Apel afirma que «incluso las ciencias naturales "explicativas" presuponen en sus conceptos fundamentales (como los de "materia", "masa", "energía" o "movimiento") una comprensión del mundo sacada del lenguaje cotidiano» (APEL, K. O., TF I, 111). En otro escrito, Apel reflexiona en torno a la «(...) función apriórica básica que cumple el lenguaje corriente como metalenguaje último de todas las construcciones logísticas (...)» (ApEL, K. O., TF I, 140).

33 Cf. ApEL, K. O., "Kann es ein wissenschaftliches...», op. cit.

34 Cf. Apel, K. O., «Technognomie...», op. cit., 75 [la traducción es mía]. 
lenguaje. «Tecnognomía» es el término que Apel utiliza para señalar una estructura fundamental del ser-en-el-mundo cognoscente que revela que todo conocimiento depende a priori del centro finito de la corporalidad humana ${ }^{35}$. Así, el hecho de que la lengua constituya para Apel el órgano más antiguo y fundamental de la Tecnognomía implica que en la base de todo conocimiento humano en general se halla, como condición de posibilidad del mismo, la imagen del mundo, prereflexiva, corporal y finita, abierta en el lenguaje materno.

\subsection{Lenguaje: el círculo hermenéutico entre estructura y contenido}

Ahora bien, siendo cierto que tanto el a priori de la reflexión como el a priori corporal dependen del a priori lingüístico, Apel considera que el propio lenguaje, que introduce la perspectiva corporal y finita en la reflexión, permanece siempre abierto a esta, hecho que permite ir renovando la perspectiva desde la cual llevar a cabo nuevas reflexiones. No obstante, Apel recuerda que «la reflexión siempre está en disposición de superar las limitaciones del a priori corporal, aunque una superación completa no se realizará jamás» ${ }^{36}$, pues toda reflexión se lleva a cabo por medio del lenguaje humano. En definitiva, se pueden renovar las perspectivas desde las cuales reflexionar pero no es posible prescindir de ellas.

La idea de que el lenguaje permanece abierto a la reflexión conecta con las afirmaciones que Apel realiza acerca del círculo hermenéutico que existe entre la forma y el contenido del lenguaje humano, entendiendo por «forma» la estructura sintáctico-semántica y por «contenido» la imagen del mundo que dicha forma lingüística abre en el trato cotidiano con las cosas. En relación a esto, Apel afirma que estructura y contenido del lenguaje «están una con otra en la relación del círculo hermenéutico, es decir, que se corrigen mutuamente de modo incesante (...)» ${ }^{37} \mathrm{y}$ que «esta situación primordial del círculo hermenéutico no puede indudablemente borrar la polaridad, con todo persistente, de la forma y el contenido, del orden universalmente válido y la vivencia instalada en una perspectiva parcial, de la reflexión excéntrica y el compromiso práctico-corporal con el mundo (...)» ${ }^{38}$. Aquí se observa cómo Apel identifica la forma del lenguaje con el a priori de la reflexión, y el contenido del mismo con el a priori corporal. Esa identificación va a jugar un papel fundamental en la concepción sintética ${ }^{39}$ del a priori del lenguaje propuesta en la reconstrucción que este trabajo ofrece.

35 Mediante esta categoría gnoseo-antropológica, Apel no hace sino destacar una particularidad propia del conocimiento humano que ha sido tradicionalmente olvidada o denostada por la gnoseología clásica: que la corporalidad, lejos de constituir un obstáculo, no es sino una condición de posibilidad para que las cosas se muestren tal y como ellas son «en sí» (cf. APEL, K. O., «Technognomie...», op. cit.). Para entender la relación entre el $a$ priori corporal y la Tecnognomía, Centeno define el primero como la estructura ontológicoexistencial que hace referencia a la finitud de la condición humana y la segunda como la concreta configuración óntico-existentiva del a priori corporal (CENTENO, A., op. cit., 39-41).

36 Apel, K. O., "“A priori de la facticidad”...», op. cit., 270.

37 APEL, K. O., TF I, 180.

38 ApEL, K. O., TF I, 186.

39 Esta expresión es utilizada por el propio Apel cuando habla del lenguaje como «fundamento del conocimiento creador en el sentido de un a priori sintético» (APEL, K. O., TF I, 183). 
3. El epílogo de 2005 a la luZ de la ENTREVISTA DE 1991 y de los ESCRITos GNOSEO-ANTROPOLÓGICOS

En su epílogo de 2005, Apel afirma, por un lado, que su pragmática trascendental del lenguaje se distingue del idealismo trascendental de Kant, que toma como punto de partida el a priori de la conciencia, porque tiene en cuenta un a priori del cuerpo colectivo del conocimiento, dada la vinculación existente entre el pensamiento y el lenguaje $\mathrm{e}^{40}$. Por otro lado, en conexión con esta idea, el autor afirma que «el a priori del lenguaje del conocimiento no atañe solo al a priori céntrico del cuerpo (...), sino igualmente al a priori excéntrico de la reflexión. Si bien las teorías reflexivamente mediadas de la ciencia pueden distanciar o relativizar el a priori céntrico del cuerpo (...), no podemos trascender el a priori del lenguaje del conocimiento gracias a la reflexión. Al contrario, solo el a priori del lenguaje permite atribuir una posible validez intersubjetiva a toda reflexión teórica ${ }^{41}$.

Lo que sigue está dedicado a analizar las ideas contenidas en este epílogo, para cuyo desarrollo e interpretación se tomarán como referencia las reflexiones llevadas a cabo anteriormente en conjunción con otras pertenecientes a los escritos gnoseo-antropológicos de Apel.

\subsection{La irrebasabilidad del lenguaje frente al cuerpo y la reflexión}

En estas reflexiones es posible distinguir varias tesis importantes. En primer lugar, la pragmática trascendental del lenguaje, que presupone la conexión que ya siempre existe entre pensamiento y lenguaje, es decir, que parte de la base de que toda reflexión llevada a cabo por la conciencia se encuentra ya siempre mediada lingüísticamente, tiene que reconocer, junto con el a priori de la reflexión, el a priori del cuerpo. En este punto es posible comprobar cómo aquí, de manera indirecta, Apel establece una relación entre cuerpo y lenguaje que recuerda en cierto modo a aquella afirmación realizada en su entrevista de 1991, según la cual para la gnoseoantropología el a priori corporal se oculta en el lenguaje. Además, se observa cómo, en este caso, el tradicional a priori del cuerpo es redefinido como «a priori del cuerpo colectivo", si bien es cierto que esta noción del a priori corporal podría estar ya en germen en sus escritos gnoseo-antropológicos, cuando Apel afirma que «en el lenguaje, en su dimensión de uso lingüístico, no solo tiene lugar la mediación de la praxis corporal del pensamiento sino también la mediación de la praxis conductual de un grupo social humano» ${ }^{42}$.

\footnotetext{
40 Apel, K. O., «Postface trente-huit ans après», op. cit., 95 [la traducción es mía].

41 Ibid., 96.

42 ApeL, K. O., TF II, 23. La concepción colectiva del cuerpo presente en la pragmática trascendental del lenguaje parecería pasar por alto la individualidad corporal de cada seren-el-mundo. Este hecho se ve mucho más claro cuando Apel vincula el a priori del cuerpo colectivo con la comunidad real de comunicación (ver infra).

En relación a la concepción lingüística de la corporalidad, Apel sostiene una importante afirmación: "la estructura del lenguaje materno acompaña, pues, de alguna manera al individuo en sus vivencias. De hecho todas las "vivencias" del hombre son ya en germen (...) "actos de comprensión", lo cual quiere decir que las vivencias se hallan constituidas en el
} 
En segundo lugar, Apel afirma que el a priori del lenguaje no solo atañe al $a$ priori corporal, en el sentido de que introduce una perspectiva corporal, haciendo de este modo posible todo tipo de reflexión en general, sino también al a priori de la reflexión, en el sentido de que gracias al lenguaje es posible llevar a cabo reflexiones válidas intersubjetivamente. La primera parte de esta afirmación recuerda a aquella otra de su entrevista de 1991, según la cual, «tanto el a priori de la reflexión como el a priori corporal pertenecen al a priori lingüístico del conocimiento ${ }^{43}$, entendiendo esa relación de pertenencia como anteriormente se propuso: como una relación de dependencia del cuerpo y de la reflexión con respecto al lenguaje. La interpretación a favor de esta propuesta obtiene asimismo su apoyo en la tesis de la «irrebasabilidad» (hintergehbar) del lenguaje defendida por Apel en el epílogo de 2005, en virtud de la cual ninguna teoría del conocimiento legítima puede obviar el presupuesto trascendental de la comunicación lingüística ${ }^{44}$.

Finalmente, la idea de que el a priori del lenguaje es el único garante de la validez intersubjetiva de nuestras reflexiones se encuentra también en la tesis apeliana de que «toda lengua materna arraiga en el logos universal, lo cual hace posible la comunicación humana, la traducción entre lenguas y la lingüística comparada ${ }^{45}$, o en la relativa a la existencia de un «(...) orden universalmente válido inmanente al lenguaje, orden siempre de carácter público (...)» ${ }^{46}$. De este modo, el lenguaje, en virtud de esa doble relación que guarda con el aspecto corporal y la dimensión reflexiva del conocimiento, no solo es condición a priori de posibilidad de la constitución del sentido del mundo sino también de la justificación de la validez intersubjetiva de los enunciados.

lenguaje y con vistas a hacerse "públicas" en el lenguaje. Esto vale incluso para el caso límite de las llamadas sensaciones (...)» (APEL, K. O., TF I, 118). Sin embargo, cabría plantearse si en el ámbito de la constitución de sentido intervienen además otros elementos no lingüísticos. En relación al idealismo lingüístico en la tradición hermenéutica alemana y, en concreto, en Apel, véanse los trabajos de C. Lafont y G. Scivoletto, respectivamente (LAFonT, C., Lenguaje y apertura del mundo: el giro lingüístico de la hermenéutica de Heidegger, Alianza Editorial, Madrid, 1997; id., La razón como lenguaje. Una revisión del "giro lingüístico» en la filosofía del lenguaje alemana, Visor, Madrid, 2002; Scivoletto, G., «Signo y lenguaje: Acerca de la interpretación de Apel y Lafont de Ser y tiempo», en: Tópicos, Revista de filosofía, 22, 2011, 231-48 e id., "Lenguaje y realidad: notas en torno al idealismo lingüístico en Habermas y Apel», en: Di Silvestre, C. et al. (eds.), Modelos de comprensión del lenguaje en la filosofía contemporánea, Guaymallen, Qellqasqa, 2016, 81-107.

43 Apel, K. O., «"A priori de la facticidad”...», op. cit., 270.

44 Cf. ApEL, K. O., "Postface trente-huit ans après», op. cit., 96 y TF II, 301. De acuerdo con Apel, la gnoseo-antropología (al igual que la pragmática trascendental del lenguaje) es una teoría del conocimiento que parte de la función de la comunicación lingüística o de la comunidad de comunicación (cf. ApEL, K. O., «Die erkenntnisanthropologische Funktion...», op. cit.). Por este motivo, y frente a la opinión de Centeno, la antropología del conocimiento sí asume el giro lingüístico.

45 APEL, K. O., TF I, 126.

46 APEL, K. O., TF I, 186. 


\subsection{Integración dialéctica de cuerpo y reflexión en la noción de "comunidad de comunicación»}

Ahora bien, las reflexiones en torno a la conexión entre la antropología del conocimiento y la pragmática trascendental del lenguaje no terminan aquí y, queriendo insistir en el vínculo que une a ambos programas, Apel se esfuerza por mostrar esta conexión de otro modo. Así, considera el autor en este mismo escrito, la pragmática trascendental del lenguaje permite superar el solipsismo metodológico del a priori de la conciencia en virtud de un a priori de la comunidad de comunicación que, a diferencia del a priori de la conciencia y al igual que el $a$ priori del lenguaje, posee una doble estructura dialéctica:

(...) En tanto que «a priori del cuerpo colectivo», remite a la presuposición de una comunidad de comunicación real, caracterizada por una lengua y una cultura particular, que corresponde a la «facticidad» y a la «historicidad» [Geschichtlichkeit] del ser-en-el-mundo en el sentido de Heidegger y Gadamer. (...) En tanto que «a priori del discurso argumentativo», que representa la forma de reflexión irrebasable [nicht hintergehbar] de toda comunicación, remite a la presuposición de una comunidad de comunicación ilimitada e ideal (...) ya siempre presupuesta por las pretensiones de validez universales [Geltungsansprüche] de toda argumentación (...). ${ }^{47}$

Llegados a este punto, es posible llevar a cabo tres observaciones. En primer lugar, vemos de qué manera el a priori de la reflexión y el a priori corporal, que en el programa gnoseo-antropológico desempeñan un papel fundamental en tanto que condiciones de posibilidad del conocimiento, son integrados en el programa pragmático-trascendental a través del a priori de la comunidad de comunicación en virtud de la doble estructura dialéctica que este a priori presenta.

En segundo lugar, se observa que los a priori del lenguaje y de la reflexión, al igual que ocurre con el a priori corporal, obtienen una nueva definición en el marco de la pragmática trascendental del lenguaje. Por un lado, la concepción del a priori del lenguaje como a priori de la comunidad de comunicación se encuentra ya en germen en la antropología del conocimiento, cuando Apel habla de la comunidad de comunicación como presupuesto del que parte su teoría del conocimiento ${ }^{48}$. Además, la «doble estructura dialéctica» que presenta el a priori de la comunidad de comunicación en el programa pragmático-trascendental coincide con la «naturaleza sintética» del a priori del lenguaje en su programa gnoseo-antropológico. Por otro lado, la concepción del $a$ priori de la reflexión como a priori del discurso argumentativo posee ya en cierto modo un antecedente en la antropología del conocimiento cuando Apel concibe la discusión dialéctica o el diálogo racional como institución última de la reflexión ${ }^{49}$.

Finalmente, cabe decir que la conexión que Apel establece en la pragmática trascendental del lenguaje entre el a priori del cuerpo y la comunidad real de comunicación, por un lado, y entre el a priori de la reflexión y la comunidad ideal de comunicación, por otro, no tiene lugar en la antropología del conocimiento, si bien es cierto que en este programa se habla ya de la comunidad ilimitada de comunicación como un a priori del conocimiento ${ }^{50}$.

48 Cf. APEL, K. O., «Die erkenntnisanthropologische Funktion...», op. cit.

49 Cf. ApEL, K. O., TF I, 191-214.

50 Cf. Apel, K. O., «Die erkenntnisanthropologische Funktion...», op. cit. 


\subsection{El triunfo pragmático-trascendental de la reflexión frente a la primacía gnoseo- antropológica del cuerpo}

Con respecto a la relación que existe entre estos tres a priori del conocimiento, pareciera que, en el marco de la pragmática trascendental del lenguaje, tanto el $a$ priori del cuerpo como el a priori de la reflexión pertenecen al a priori del lenguaje, tal y como Apel afirma en su entrevista de 1991 en el marco de la gnoseo-antropología. En relación a esta cuestión, Apel lleva a cabo una importante reflexión al final de su epílogo.

De la lectura de su escrito Das Leibapriori der Erkenntnis (1963) parece inferirse la idea de que una superación completa del a priori corporal por parte del a priori de la reflexión no se realizará jamás. De hecho, Apel afirma en relación a esto lo siguiente: "ni siquiera hoy en día espero en modo alguno retirar la tesis gnoseoantropológica que está implicada $»^{51}$. Ahora bien, Apel reconoce que sosteniendo esta tesis da la impresión de estar negando la importancia de la reflexión filosófica en torno a valores y normas y menospreciando su función orientativa en el ámbito de la praxis individual y colectiva. De acuerdo con el autor, «si bien la reflexión filosófica no permite realizar una supresión objetivante de las perspectivas constitutivas del sentido del compromiso de la praxis existencial o colectiva en el seno de una teoría (...), sí que puede recurrir a los presupuestos no rebasables (...) del a priori de la argumentación ${ }^{52}$.

Así, una reflexión pragmático-trascendental en torno a los presupuestos de la argumentación, que según Apel representa la forma de reflexión irrebasable de toda comunicación, permite llevar a cabo «una fundamentación última de la filosofía que representaría el triunfo del a priori de la reflexión ${ }^{53}$. De este modo, si en la antropología del conocimiento la polaridad existente entre el a priori de la reflexión y el a priori del cuerpo se decanta más bien en favor de la primacía del cuerpo sobre la reflexión, observamos que en la pragmática trascendental del lenguaje dicha primacía la detenta el a priori de la reflexión. La diferencia entre ambos programas radicaría en la distinta concepción que de la reflexión se maneja en los mismos.

De este modo, mientras que la función de la reflexión gnoseo-antropológica ${ }^{54}$ no es otra que asumir u objetivar las condiciones que hacen posible el conocimiento de cara a elaborar teorías válidas intersubjetivamente, la reflexión pragmáticotrascendental tematiza tales condiciones con vistas a una fundamentación última

51 APEL, K. O., «Postface trente-huit ans après», op. cit., 98 [la traducción es mía]. Tal y como se pudo ver, Apel también afirma en la entrevista de 1991 que «la reflexión siempre está en disposición de superar las limitaciones del a priori corporal, aunque una superación completa no se realizará jamás» (op. cit., 270), pues toda reflexión se lleva a cabo por medio del lenguaje humano. En definitiva, se pueden renovar las perspectivas desde las cuales reflexionar pero resulta imposible prescindir de ellas.

52 ApeL, K. O., «Postface trente-huit ans après», op. cit., 100 [la traducción es mía]. En el marco de la pragmática trascendental del lenguaje, lo que caracteriza a estos presupuestos frente a otros es el hecho de que no pueden ser cuestionados sin auto-contradicción performativa ni deducidos sin petición de principio.

${ }^{53}$ APEL, K. O., «Postface trente-huit ans après», op. cit., 101[la traducción es mía].

54 A favor del tratamiento diferenciado de la reflexión en estos dos programas se muestra también Centeno (op. cit., 34). 
de la filosofía ${ }^{55}$. A favor de esta hipótesis hablaría la sugerencia realizada por Apel de que la pragmática trascendental del lenguaje vendría a resolver el problema relativo a la legitimación filosófico-trascendental de la antropología del conocimiento: «dicha concepción (la de la pragmática trascendental) me dio la posibilidad de integrar la mencionada transformación gnoseo-antropológica de la filosofía trascendental y de dotarla de una base trascendental que no pudiera ser tachada de psicologismo o naturalismo ${ }^{56}$.

4. El a PRIORI GNOSEO-ANTROPOLÓGICO DEL LENGUAJE REINTERPRETADO A PARTIR DEL A PRIORI PRAGMÁTICO-TRASCENDENTAL DE LA COMUNIDAD DE COMUNICACIÓN

Para finalizar esta reconstrucción de la problemática de los a priori del conocimiento, cabe presentar la ya mencionada naturaleza sintética del a priori del lenguaje en relación a los otros dos a priori gnoseo-antropológicos, siguiendo así el modo de proceder de Apel cuando reflexiona en torno a la doble estructura dialéctica del a priori de la comunidad de comunicación.

Así, en la antropología del conocimiento, el a priori del lenguaje posee una naturaleza sintética:

1) En tanto «a priori del cuerpo $»^{57}$, remite al contenido o imagen del mundo (pre-reflexiva y céntrica) abierta en la lengua materna y es condición a priori de posibilidad de la constitución del sentido. En el contexto de la tensión polar que existe entre el cuerpo y la reflexión, el primero tiene primacía con respecto a la segunda en el marco de la experiencia humana.

2) En tanto que «a priori de la reflexión», remite al orden o forma del mundo (reflexiva y excéntrica) propia del logos universal y es condición a priori de posibilidad de la justificación de la validez intersubjetiva de nuestro conocimiento. En el contexto de la tensión polar que existe entre el cuerpo y la reflexión, esta última tiene primacía con respecto al primero en el marco de las construcciones teóricas.

A favor de esta concepción sintética del a priori lingüístico y su conexión con el programa pragmático-trascendental habla la siguiente consideración realizada por Apel:

¿Existe alguna instancia común —en el sentido de las condiciones trascendentales de significación - que, por un lado, esté arraigada existencialmente en cada

55 En este punto se plantean varias cuestiones: esta reflexión pragmático-trascendental en torno a los presupuestos de la argumentación, en la medida en que se halla mediada lingüísticamente, ¿no seguiría en última instancia dependiendo de una perspectiva corporal? ¿Desde qué perspectiva corporal se lleva a cabo esta reflexión pragmático-trascendental para que sea válida intersubjetivamente?

56 Apel, K. O., "Transformation der Transzendentalphilosophie: Versuch einer retrospektiven Zwischenbilanz», en: Mercier, A. (ed.), Philosophes critiques d'eux-mêmes, 4, Peter Lang, Berna / Frankfurt / Las Vegas, 1978, 19-20 [la traducción es mía].

57 Este a priori gnoseo-antropológico integraría los órganos sensoriales, los instrumentos técnicos y los intereses del conocimiento (cf. ApEL, K. O., TF II, 91-120). 
sujeto singular, finito y viviente capaz de comprensión y que, por otro lado, en tanto condición de posibilidad de la validez intersubjetiva del sentido, trascienda necesariamente sin embargo toda individualización y finitud? Creo que se debe responder afirmativamente a esa pregunta. La instancia buscada es el lenguaje humano, si se lo considera un a priori, no solo bajo las «condiciones existenciarias de posibilidad de la constitución de la significación» sino también, por otra parte, bajo las «condiciones de posibilidad de la validez intersubjetiva de la significación» no reducibles a condiciones lógico-semánticas. La disciplina filosófica que habría que proponer para ese fin se debería llamar, a mi modo de ver, «pragmática trascendental del lenguaje» (...). ${ }^{58}$

Esta reinterpretación de la relación existente entre los a priori gnoseoantropológicos del cuerpo, la reflexión y el lenguaje, tomando como referencia el a priori pragmático-trascendental de la comunidad de comunicación, abre una posible vía de integración entre el programa de la antropología del conocimiento y el de la pragmática trascendental del lenguaje.

\section{CONCLUSIONES}

En este apartado se recogen de manera sucinta las principales conclusiones a las que se ha llegado a lo largo de este trabajo:

1) Apel conecta la antropología del conocimiento con la pragmática trascendental del lenguaje por medio de los a priori del conocimiento.

2) El a priori corporal y el a priori de la reflexión, que en el programa gnoseoantropológico desempeñan un papel fundamental en tanto que condiciones de posibilidad del conocimiento, son integrados en el programa pragmáticotrascendental a través del a priori de la comunidad de comunicación, en virtud de la doble estructura dialéctica que este a priori presenta.

3) En la pragmática trascendental del lenguaje, el a priori del lenguaje de la antropología del conocimiento es redefinido como a priori de la comunidad de comunicación, el del cuerpo como a priori del cuerpo colectivo y el de la reflexión como a priori del discurso argumentativo. Ahora bien, tales nociones se encuentran ya en germen en el programa gnoseo-antropológico.

4) La conexión que Apel establece en la pragmática trascendental entre el $a$ priori del cuerpo y la comunidad real de comunicación, por un lado, y entre el a priori de la reflexión y la comunidad ideal de comunicación, por otro, no tiene lugar en la antropología del conocimiento, a pesar de que en este último programa se hablara ya de la comunidad ilimitada de comunicación como un a priori del conocimiento.

5) Aunque en el programa gnoseo-antropológico se da una polaridad entre el $a$ priori del cuerpo y el a priori de la reflexión, Apel hace depender en última instancia el a priori de la reflexión del a priori corporal. En cambio, afirma que la fundamentación última posibilitada por el programa pragmáticotrascendental constituye un triunfo del a priori de la reflexión sobre el $a$

58 ApeL, K. O., «¿Es la muerte una condición...», op. cit., 93. 
priori corporal. En ambos programas, los momentos del a priori reflexivo y del a priori corporal pertenecen y dependen del a priori del lenguaje y de la comunicación.

6) En cuanto a la reflexión, esta no es tratada en la antropología del conocimiento en su vertiente pragmático-trascendental, a diferencia de lo que ocurre en su programa posterior.

7) La naturaleza del a priori gnoseo-antropológico del lenguaje es sintética, en el sentido de que permite introducir una perspectiva corporal que constituye el sentido y, a la vez, hace posible justificar la validez intersubjetiva del conocimiento. De este modo, este a priori presenta una naturaleza idéntica a la doble estructura dialéctica que caracteriza el a priori pragmáticotrascendental de la comunidad de comunicación, ya que incorpora los a priori gnoseo-antropológicos del cuerpo y de la reflexión.

Universidad de Granada (España)

Laura Molina-Molina

Departamento Filosofía II. Facultad de Filosofía y Letras.

Rheinische Friedrich - Wilhelms - Universität Bonn (Alemania)

Internationales Zentrum für Philosophie NRW (IZPH)

lauramolina@ugr.es

[Artículo aprobado para publicación en enero de 2019] 